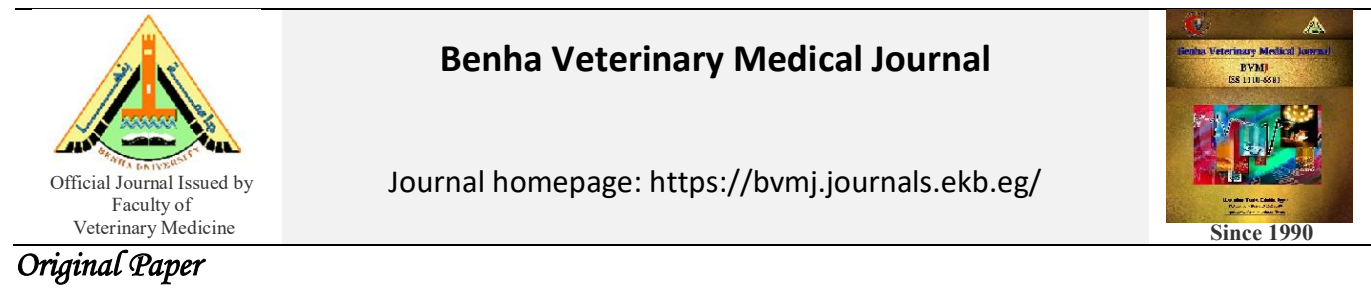

\title{
Therapeutic and protective role of curcumin nanoparticles against experimentally induced hepatotoxicity in rats
}

Afaf Desouky Abdelmgid; Hussien Abdelmaqsud Ali; and Marwa A. Ahmed*.

Department of Biochemistry, Faculty of Veterinary Medicine, Benha University, Egypt

\begin{tabular}{l} 
ARTICLE INFO \\
\hline Keywords \\
Cattle \\
FMDV \\
NSP \\
ELISA kit \\
MPV \\
\hline Received 27/03/2021 \\
Accepted $01 / 04 / 2021$ \\
Availa6le On-Line \\
01/07/2021
\end{tabular}

\begin{abstract}
The goal of this research was to see if using Cur-NPs could boost curcumin bioavailability and to see whether Cur-NPs could protect rats from $\mathrm{Cp}$-induced hepatotoxicity. Five groups of fifty white albino rats were formed at random. Group I acted as negative control while group II received Cyclophosphamide daily dosage of $(5 \mathrm{mg} / \mathrm{kg}$ body weight) via oral gavage for 8 weeks. Group III received Cur-NPs at a dosage of $(20 \mathrm{mg} / \mathrm{kg}$ bw $) 3$ days a week via oral gavage for 4 weeks. Group IV received CP as group II and treated with Cur-NPs as group III for 4 weeks. Group V received Cur-NPs as protector, for 4 weeks before $\mathrm{CP}$ administration as group II with continuous administration with Cur-NPs till the end. At the end of the experiment, blood and tissue samples were obtained for biochemical, antioxidant enzyme, and cytokine level determination. According to the findings, giving CP to rats in group II increased serum liver function enzymes (ALT, AST, and ALP), which was followed by a substantial decrease in serum albumin conc. and tissue antioxidants (GPx, SOD, and CAT) activity, as well as increased levels of cytokines (IL-6, IL-1 $\beta$ ). Cur-NPs treatment improved these biochemical changes dramatically, particularly in group These findings suggest that Cur-NPs may be a potential hepatoprotective agent for reducing liver oxidative stress caused by various stress factors.
\end{abstract}

\section{INTRODUCTION}

The liver is crucial to maintaining body homeostasis. It is concerned within the majority of all the biochemical pathways of growth, immunity, nutrient supply and in providing energy. So, maintaining healthy liver is a crucial issue for the overall health (Muhammed et al., 2021). Drug hepatotoxicity is the injury or damage to the liver caused by drug exposure; it is a potentially fatal adverse drug reaction. Hepatotoxicity can be classified as hepatocellular, cholestatic, or mixed, depending on how much alanine aminotransferase and alkaline phosphatase activity rises above normal levels. Alcohol consumption, idiosyncrasy, gender, age, occurrence when taking other medications, prior or underlying liver disease, genetic and environmental factors are all risk factors. Nonspecific symptoms such as abdominal pain, jaundice, fever, diarrhea, vomiting, nausea, pruritus, and rash are commonly associated with liver toxicity manifestations (Paniagua and Amariles, 2017).

Cyclophosphamide is a drug used as an anti-cancer drug which is a chemotherapeutic and immunosuppressive drug to treat wide range of neoplastic as well as some autoimmune diseases. Cyclophosphamide adds an alkyl group to DNA guanine bases due to its alkylating antitumor agent properties, inducing the synthesis of aberrant cytosinethymine couples and forcing the cells' DNA reparation mechanism to eliminate the modified guanine, resulting in cell apoptosis. (Matalon et al., 2004; Altaylý et al., 2012). $\mathrm{CP}$, an alkylating chemotherapeutic drug, is metabolized by liver cytochrome P450 enzymes, namely CYP3A4 and
CYP2B6 which demonstrate active therapeutic and cytotoxic metabolites and diffuse out of the hepatocytes into the plasma (Yildiz, et al., 2019). CP spreads throughout the body and generates two active metabolites: phosphoramide mustard (PAM) and acrolein (ACR). While CP's immunosuppressive and antineoplastic effects are related with PAM, ACR is responsible for its undesirable toxicity. ACR produces highly reactive oxygen species (ROS) and oxidative stress in hepatocytes (Mohammad, et al., 2012, MacAllister, et al., 2013). Thus, it interacts with protein and causes changes in the structure and function of enzymes and harms the tissue antioxidant defense mechanism. CPinduced toxicity is a consequence of a mitochondrial dysfunction and ends with a decrease of adenosine triphosphate owing to nitrosative and oxidative stress (Yildiz, et al., 2019).

Curcumin's biological activity is attributed to its bioavailability in humans. The volume and concentration at which curcumin is engrossed, occurs in the plasma, and reaches its target position have all been addressed in bioavailability studies. Curcumin has been studied for over three decades for its anti-tumoral, anti-inflammatory, antimutagenic, antioxidant, antimicrobial, wound healing, and anti-angiogenesis properties (Imran et al., 2018; Willenbacher et al., 2019). Curcumin has been shown to have beneficial effects on a number of human diseases, like tumors (Adiwidjaja et al., 2017), liver disorders (Nabavi et al., 2014), and certain other inflammatory disorders (Beevers and Huang, 2011). Even after its well-documented advantages, curcumin's usefulness is usually limited by

*Corresponding author: dr.marwa_lab@yahoo.com 
some factors. For example, low pharmacokinetics and bioavailability, weak bioactive absorption, rapid metabolization, low penetration and targeting efficacy, sensitivity to alkaline environments, metal ions, light, and heat are all factors to be considered. (Flora et al., 2013).

These obstacles, however, are being overcome by encapsulating curcumin in nanoformulations nanocurcumin (Yallapu et al., 2012). Nanoparticles are particles of approximately $1-100 \mathrm{~nm}$ in diameter possess unique physical, chemical, and biological properties that can be useful for drug delivery (Biswas et al., 2014). Nanoparticles are 1,000 times smaller than the average human body cell and consist of materials engineered at the atomic or molecular level. They are also suitable for both controlled and targeted drug delivery systems (Rudramurthy et al., 2016). Encapsulating drugs inside nanoparticles can enhance the pharmacokinetics and solubility of drugs; provide targeted delivery and controlled release of drugs. So far, polymer, solid lipid, magnetic, gold, and albumin-based nanoparticles are extensively used to improve the curcumin therapeutic applications. Implementing curcumin into nanoparticles using a variety of techniques is an acceptable and successful choice for improving curcumin's biological activity, raising its bioavailability and solubility, allowing for long-term circulation and retention in the body, and avoiding curcumin's physiological barriers (Fonseca-Santos et al., 2016). By spreading the indent tissues, it can also reduce unintended toxicity to adjacent normal cells/tissues. The study was designed to reveal the ameliorative properties of Cur-NPs on hepatic toxicity induced experimentally in male albino rats by using Cyclophosphamide.

\section{MATERIAL AND METHODS}

one hundred and sixty sera were obtained from periodically 2.1. Experimental animals:

Fifty white male albino rats, aged 10-12 weeks and weighing 140-160 g, were used in this work. Rats were collected from the laboratory animal research unit, Moshtohor, Benha University's faculty of veterinary medicine. Rats were housed in normal light and temperature environments, with free access to a standard pellet diet containing $21 \%$ protein and ad-libitum tap water. Prior to the experiment, the animals were given ten days to acclimate.

\subsection{Chemicals:}

Chemicals used were analytical grade and came from wellknown commercial sources. In this work, the following chemicals were used:

2.2.1. Commercially available CP tablets (Endoxan ${ }^{\circledR} 50 \mathrm{mg}$, Baxter Oncology GmbH).

Preparation of Cyclophosphamide (CP): The accurate doses of the drugs were dissolved in saline solution daily and shortly before administration. ( $5 \mathrm{mg} / \mathrm{kg}$ body weight) daily by gavage for 8 weeks.

2.2.2. Curcumin was purchased from Sigma Chemical Co. (St. Louis, MO, USA.)

2.2.3 Synthesis of nano-curcumin:

To prepare curcumin nanoparticles, $1 \mathrm{M}$ curcumin with low solubility in water was combined with a $4 \mathrm{M}$ sodium bicarbonate buffer, which was then ground for 8 hours in a mechanical ball mill (350 rounds per second). The nanorange of the novel nanocomposite (Basic curcumin) was depicted by a TEM (Transmission electron microscope) image to appear in spherical shape with a diameter of 15 $62.7 \mathrm{~nm}$.

Preparation and dosage of nano-curcumin: Nano-Cur was freshly dissolved in distilled water, dose level given to rats was $(20 \mathrm{mg} / \mathrm{kg} \mathrm{b.wt)}$ via oral gavage daily, (Hassan et al., 2014).

\subsection{Experimental Design:}

Five separate groups were divided at random, 10 rats per each:

2.3.1 Group (1): Negative control:

Rats were fed a standard diet without any medication for the duration of the study.

2.3.2 Group (2): Cyclophosphamide group "Positive group": Rats received CP at a dose of ( $5 \mathrm{mg} / \mathrm{kg}$ body weight) daily by gavage for 8 weeks (Gad El-Karim and El-Amrawi (2019).

\subsubsection{Group (3): NanoCurcumin group:}

Rats received Cur-NPs ( $20 \mathrm{mg} / \mathrm{kg}$ body weight) orally three times per week by gavage for 12 weeks (Hassan et al., 2014). 2.3.4 Group (4): Cyclophosphamide + NanoCurcumin group:

Firstly, rats received CP as group (2) for 8 weeks, followed by receiving Cur-NPs as group (3) for 4 weeks.

2.3.5 Group (5): NanoCurcumin group + Cyclophosphamide:

Firstly, rats received Cur-NPs as group (3) for 4 weeks as protector then, followed by $\mathrm{CP}$ as group (2) for 8 weeks with continuous administration of Cur-NPs.

\subsection{Sampling:}

Each group of rats was fasted overnight and then euthanized after 12 weeks. At the end of the experiment, blood samples and liver tissue specimens were obtained from all groups.

2.4.1 Blood samples:

Blood samples were obtained from retro-orbital plexus of the eyes puncture. Blood was left to clot then centrifuged for 15 minutes at 3000r.p.m. Sera were separated in dry sterile tubes by automatic pipette, and then stored at $-20{ }^{\circ} \mathrm{C}$ in a deep freezer until determination of biochemical parameters. 2.4.2Tissue sample:

The liver was easily removed, washed in ice cold saline, snap-frozen in liquid nitrogen, and deposited at $-80^{\circ} \mathrm{C}$.

One gram of liver tissue was cut and minced into small pieces, then homogenised with a glass homogenizer in 9 volumes of ice-cold $0.05 \mathrm{mM}$ potassium phosphate buffer (pH7.4) to make $10 \%$ homogenates, then centrifuged for 15 minutes at 6000 r.p.m. at $4{ }^{\circ} \mathrm{C}$. The supernatant antioxidant activity was measured immediately.

\subsection{Analysis:}

2.5.1 Biochemical analysis:

Liver enzymes activity alanine aminotransferase (ALT), aspartate amino transferase (AST), Alkaline Phosphatase (ALP) were determined in the serum using human diagnostics worldwide kits (Germany), also Albumin level was determined in the serum using diagnostic kit supplied by (Diamond, Egypt) according to Fischbach, et al., (1992), Klin, (1970) and Gendler, (1984) respectively.

2.5.2 Antioxidant estimation:

The supernatant of hepatic tissue homogenate was used for the determination of Catalase (CAT), Superoxide dismutase (SOD) activities, and Glutathione peroxidase (GPx) level, using biodiagnostic kit (Cairo, Egypt) according to 
Fossati.et al., (1980), Nishikimi et al., 1972 and (Paglia and Valentine et al.,1967) respectively.

2.5.3 Cytokines estimation:

IL-6 was measured by using Rat IL-6 Immunoassay, qantikine Elisa kit, (USA), Rat IL-1 beta ELISA Kit, Ray Biotech (USA) according to (Hirano, T. 1998) and (Auron et al., 1984) respectively.

2.6. Statistical analysis:

The SPSS (version 19) was used to analyze all of the gathered data. The least significant difference (LSD), Duncan as showed in tables $(1,2)$ test was used to distinguish variations between group means, and the variance of oneway analysis (ANOVA) was used to test hypotheses. The minimum level of significance was set at P-values less than 0.05 .

\section{RESULTS}

3.1 Biochemical results:

Cyclophosphamide administration to normal rats for 8 weeks resulted in a significant increase in serum ALT, AST and ALP activities when compared with negative control group. Such elevation was reduced in Cur-NPs treated group when compared with CP group. Serum Albumin showed significant decrease in $\mathrm{CP}$ treated group when compared to negative control such decreased improved significantly in protected and treated Cur-NPs groups. These data illustrated in figures 1 and 2.

3.2 Hepatic Antioxidant enzyme activities and Cytokines results:

$\mathrm{CP}$ administration resulted in a significant decrease in liver SOD, catalase and GPx levels when compared with negative control group. Cur-NPs administration as a treatment or as a protection leads to a significant increase in liver SOD and Catalase and GPx levels when compared to positive control. $\mathrm{CP}$ enhanced liver inflammation as indicated by elevated IL$1 \beta$ and IL 6 levels in group administrate CP compared with negative control. However, treatment with Cur-NPs significantly reduced levels of these inflammatory parameters in both protected and treated groups when compared with CP treated group, As shown in figures 3-6.

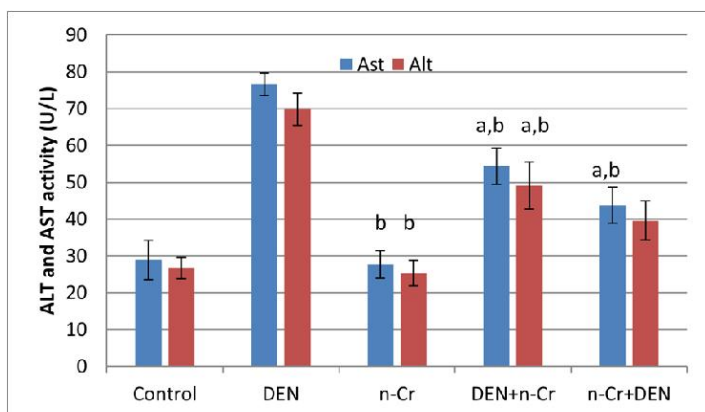

Figure 1 Serum ALT, AST activity (U/L) in control, DEN group and NanoCur treated groups of male albino rats.

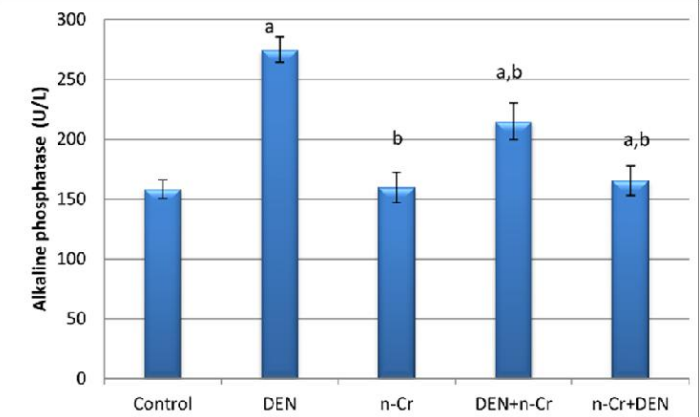

Figure 2 Serum ALP activity (U/L) in control, DEN group and Nano-Cur treated groups of male albino rats.

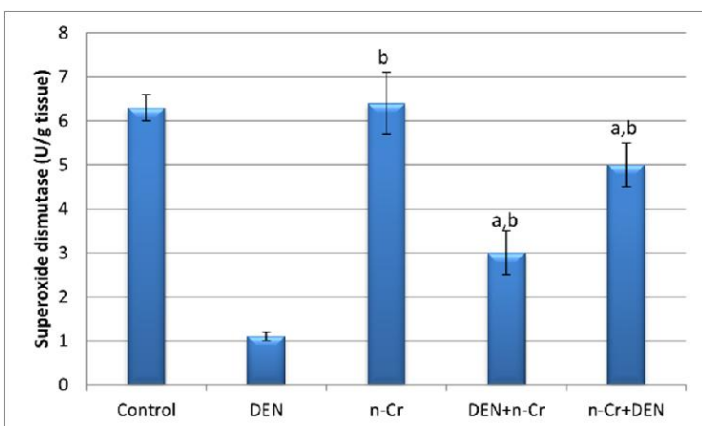

Figure 3 Hepatic SOD activity (U/g) in control, DEN group and Nano-Cur treated groups of male albino rats.

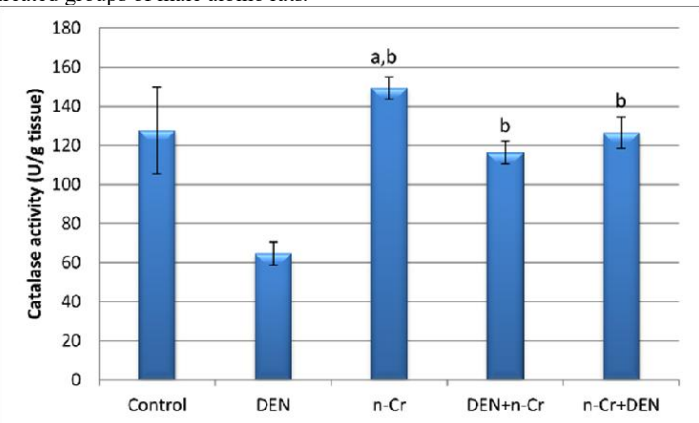

Figure 4 Hepatic CAT activity (U/g) in control, DEN group and Nano-Cur treated groups of male albino rats.

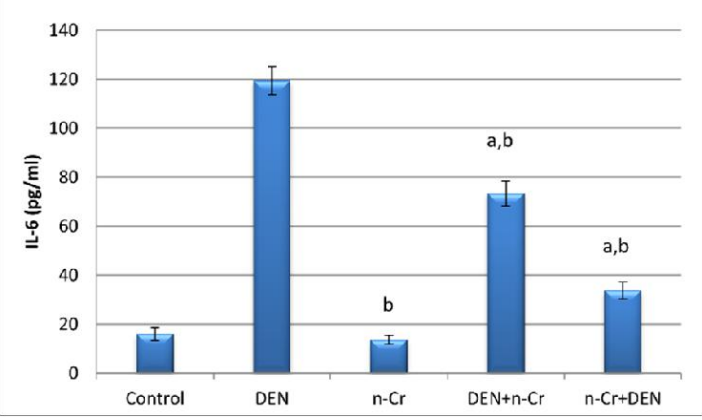

Figure 5 IL-6 activity (pg./ml) in control, DEN group and Nano-Cur treated groups of male albino rats. 


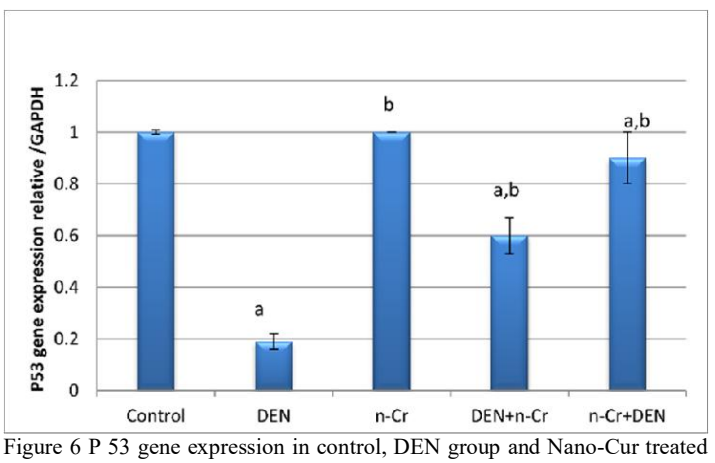

Figure 6 P 53 gene expression in control, DEN group and Nano-Cur treated groups of male albino rats.

\section{DISCUSSION}

Liver is the first major organ to detoxify either toxic chemicals or drugs. Many medications have been recorded to cause severe human hepatic damage (El-Dawy et al., 2020).

We examined the impact of Cur-NPs treatment on hepatotoxicity caused by $\mathrm{CP}$ administration in this work. Since ALT, AST and ALP are usually localized inside the cytoplasm and released into the circulation after cellular damage, a substantial increase in serum ALT, AST, and AlP levels followed by a decrease in serum albumin concentration in $\mathrm{CP}$ hepatotoxic rats indicates that hepatocyte damage has occurred (Singh et al., 2011). Damage to hepatocytes also affects their transport role and membrane permeability, resulting in enzyme leakage from the cells. (Singh et al., 2011, Singh et al., 2018) and the reduction of albumin is related discretely to liver dysfunction (Hassan et al., 2014).

Rats treated with Cur-NPs showed reduced levels of ALT, AST and ALP, such improvement reflects that Cur-NPs have the ability to protect hepatocytes from damage produced because of $\mathrm{CP}$ administration. Cur-NP treatment also restored albumin levels to normal, indicating that hepatocytes are performing well in protein synthesis.

Cyclophosphamide is an anti-cancer chemotherapeutic and immunosuppressive drug causing hepatic injury and toxicity because of the development of toxic reactive oxygen species and oxidative stress (ROS) (Manda and Bhatia, 2003; Motawi, et al., 2010). It has been reported that oxidative DNA damage is due to a hydro peroxide derivative of cyclophosphamide through $\mathrm{H}_{2} \mathrm{O}_{2}$ generation (Murata et al., 2004).

Therefore, our obtained data revealed that $\mathrm{CP}$ treated group show a decrease in GPx, SOD, and CAT activity when compared with the negative control group. This reduction in hepatic antioxidants was elevated in rats administrated with Cur-NPs either as treatment or as protection. Curcumin has been shown to have the capacity to scavenge free radicals. Curcumin's antioxidant activity has been revealed in biological models by many researchers. There is many scientific evidence on the capability of curcumin on living cells in trapping the free radicals like reactive nitrogen and oxygen species through several means and thus exhibiting the antioxidant property (Rafiee et al., 2019). It was demonstrated that curcumin increases the efficacy of free radicals scavenging activity related enzymes, but also produces inhibitory effects on enzymes which produce free radicals (Hewlings and Kalman (2017). Curcumin's capacity to scavenge free radicals may be due to the existence of methoxy and phenolic groups on the phenyl ring, as well as 1,3-diketone (Priyadarsini et al. 2003) composing it capable of prevent peroxidation of lipid. Lipid peroxidation can also be reduced by curcumin through increasing endogenous free radical scavenger content (Dickinson et al. 2003) and antioxidant enzymes such as GPx, CAT and SOD activities (Reddy and Lokesh 1994).

The antioxidant activities of nano-CUR have more potential than its native curcumin and in vitro cytotoxicity effect of nano-CUR was examined in Neuro2A cells suggests that nano-CUR has the potent candidate for thetreatment of chronic diseases (Moghaddasi et al., 2018). In another research, Ranjbar et al. (2020) studied the curcumin and nano-curcumin effects on the oxidant and antioxidant system on the liver mitochondria using aluminum phosphide (AIP) toxicity induced rat model. It was seen that nanocurcumin enhanced the oxidative stress factors and protected the liver against the adverse effects of AlP through the scavenging of free radicals and stabilizing the oxidative status (Ranjbar et al., 2020). Therefore, the efficiency of Cur-NPs in ameliorating peroxidation of lipid induced by $\mathrm{CP}$ in the present work might be mediated by its direct free radicals scavenging and by enhancing the antioxidant defense system and to inhibit free radicals formation, increase the endogenous antioxidant activity (Bruck et al., 2004).

Results also revealed that $\mathrm{CP}$ enhanced inflammatory damage indicated by the increase of pro-inflammatory cytokines IL- $1 \beta$ and IL- 6 serum concentrations. Toxic materials mediate inflammatory reaction through activation of macrophages and induction of pro-inflammatory cytokines (Kang et al., 2012). Data obtained from this study show that there was high significant increase in the level of IL-6and IL- $1 \beta$ in CP group when compared with the negative group. Within the present study, hepatic pro-inflammatory biomarkers, (IL-1 $\beta$ ) and (IL-6), significantly increased at $\mathrm{CP}$ treated rats due to the inflammation induced within the hepatic tissue because of $\mathrm{CP}$ inflammatory known effect. Our data agreed with Lacour et al., (2005) how documented that pre-clinical hepatotoxicity or, in certain instances, hepatotoxicity has been observed in a number of significant drug groups. Hepatotoxicity is related to both necrosis and apoptosis, which cause parenchyma death. Molecular mediators of the immune response, such as (IL-1 $\beta$ ) and (IL-6) have been linked to acute and chronic liver damage incertain studies. These cytokine networks have been linked to the xenobiotic response in the liver. During hepatic toxic injury, proinflammatory cytokines such as (IL1 $\beta$ ) and (IL6) are released into the bloodstream from both the liver and distal sites.

Cur-NPs, a well-known immune modulator, inhibit the expression of numerous of pro-inflammatory cytokines, including (IL1 $\beta$ ) and (IL6). Curcumin has been shown in several studies to reduce (IL1 $\beta$ ) and (IL6) mRNA expression and cytokine secretion in inflammatory cells, as discussed in the research of Yetkin et al. (2020). Curcumin was also found to reduce the amount of circulating (IL-6) in patients with systemic inflammation, according to a meta-analysis by Derosa et al. (2016). Curcumin is an anti-inflammatory and immunomodulatory agent that inhibits the proliferation and differentiation of inflammatory cells as well as the development of related cytokines. These studies illustrate why rats treated with Cur-NPs, either pre or post treatment, have lower levels of (IL1 $\beta$ ) and (IL6). 


\section{CONCLUSION}

Cur-NPs are very powerful free radical scavenger with antioxidant properties and the ability to prevent oxidative stress. It also inhibits inflammatory cells differentiation and proliferation, as well as the development of associated cytokines. Finally, nanocurcumin application is still in its initial phases. Its progress requires serious and committed efforts through a system of organized and scheduled trials based entirely on the goal of enhancing curcumin beneficial effects.

\section{REFERENCES}

1. Adiwidjaja, J., Mclachlan, A. J., and Boddy, A. V. 2017. Curcumin as a clinicallypromising anti-cancer agent: pharmacokinetics and drug interactions. Expert Opin.Drug Metab.Toxicol. 13, 953-972.

2. Altaylý, E., Malkoc, E., Alp, B.F. andKorkmaz, A. 2012.Prevention and treatment of cyclophosphamide and ifosfamide-induced hemorrhagic cystitis. J Mol Pathophysiol.;1: 53-62.

3. Beevers, C. S. and Huang, S. 2011. Pharmacological and clinical properties of curcumin. Bot.: Targets Ther. 1, 5-18.

4. Biswas, A. K., Islam, M. R., Choudhury, Z. S., Mostafa, A., and Kadir, M. F. 2014. Nanotechnology based approaches in cancer therapeutics. Adv. Natural Sci.: Nanosci. Nanotechnol.5, 043001.

5. Bruck, R., Ashkenazi, M., Weiss, S., Goldiner, I., Shapiro, H. andAeed, H. 2004. Prevention of liver cirrhosis in rats by curcumin. Liver International 2007; 27: 373-383.

6. Derosa, G., Maffioli, P., Simental-Mendia, L.E.andSahebkar, A. 2016. Effect of curcumin on circulating interleukin- 6 concentrations: a systematic review and meta-analysis of randomized controlled trials, Pharmacol. Res. 111.394-404.

7. Dickinson, D.A., Iles, K.E., Zhang, H., Blank, V. and Forman, H.J. 2003.Curcumin alters EpRE and AP-1 binding complexes and elevates glutamatecysteine ligase gene expression. FASEB J 17:473-475.

8. El-Dawy, K.H., Dowidar, K.A., Soliman, M.A. and Arisha, A.H. 2020. Ameliorative Effects of Nanocurcumin on Cyclophosphamide Induced Immunosuppression in Male Rats. Zag Vet J, Volume 48, Number 3, p. 228-241.

9. Fischbach, F. and Zawata, B. 1992.Klin. Lab. 38, 555561.

10. Flora, G., Gupta, D., and Tiwari, A. 2013. Nanocurcumin: a promising therapeutic advancement over native curcumin. Crit. Rev. Ther.TM Drug Carrier Syst. 30(4), 331-368.

11. Fonseca-Santos, B., Dos Santos, A. M., Rodero, C. F., Gremião, M. P. D., and Chorilli, M. 2016.Design, characterization, and biological evaluation of curcumin-loaded surfactant-based systems for topical drug delivery. Int. J. Nanomed. 11, 4553.

12. Fossati, P., et.al .1980 Clin. Chem. 26, $227-231$.

13. Gad El-Karim, D. R. and El-Amrawi, G. A. 2019. Cyclophosphamide hepatotoxicity: the role of cytochrome $\mathrm{C}$ oxidase and the possible protective effect. Slov Vet Res 2019; 56 (Suppl 22): 15-23.
14. Gendler, S.; Kaplan, A. et al., (1984): ClinChemYhe C.V Mosby Co. st Louis. Toronto. Princeton; 12681273 and 425.

15. Hassan, S.K., Mousa, A.M., Eshak, M.G., Farrag, A.H. and Badawi, A.M. 2014. Therapeutic and chemopreventive effects of nanocurcumin against diethylnitrosamine induced hepatocellular carcinoma in rats. Int J Pharm Sci.; 6:54-62.

16. Hewlings, S. and Kalman, D. 2017. Curcumin: a review of its'effects on human health. Foods 6, 92 .

17. Hirano, T. 1998. Interleukin 6 in The Cytokine Handbook, 3rd. ed. Academic Press, New York, p. 197.

18. Imran, M., Ullah, A., Saeed, F., Nadeem, M., Arshad, M. U., and Suleria, H. 2018.Cucurmin, anticancer, and antitumor perspectives: A comprehensive review. Crit. Rev. Food Sci. Nutr. 58, 1271-1293.

19. Kang, G.J., Kang, N.J., Han, S.C., Koo, D.H., Kang, H.K., Yoo, B.S. and Yoo, E.S. 2012. The Chloroform Fraction of Carpinus tschonoskii Leaves Inhibits the Production of Inflammatory Mediators in $\mathrm{HaCaT}$ Keratinocytes and RAW264.7 Macrophages. Toxicological Research, 28, 255-262.

20. Lacour, S., Gauteir, J.C., Pallardy, M. and Roberts, R.A. 2005. Cytokines as potential biomarkers of liver toxicity.Cancer biomarkers: section A of Disease markers 1(1):29-39.

21. MacAllister, S.L., Martin-Brisac, N., Lau, V., Yang, K. and O'Brien, P.J. 2013.Acrolein and chloroacetaldehyde: an examination of the cell and cellfree biomarkers of toxicity. ChemBiol Interact, 202(1), 259-266.

22. Manda, K. and Bhatia, A.L. 2003. Prophylactic action of melatonin against cyclophosphamide-induced oxidative stress in mice. Cell Biol Toxicol.;19(6): 367372.

23. Matalon, S.T., Ornoy, A. and Lishner, M. 2004. Review of the potential effects of three commonly used antineoplastic and immunosuppressive drugs (cyclophosphamide, azathioprine, doxorubicin on the embryo and placenta). Reprod Toxicol.;18(2):219-30.

24. Moghaddasi, F., Housaindokht, M. R., Darroudi, M., Bozorgmehr, M. R., and Sadeghi, A. 2018.Synthesis of nanocurcumin using black pepper oil by $\mathrm{O} / \mathrm{W}$ Nanoemulsion Technique and investigation of their biological activities. LWT92, 92-100.

25. Mohammad, M.K., Avila, D., Zhang, J.,Barve, S.,Arteel, G., McClain, C. and Joshi-Barve, S.2012.Acrolein cytotoxicity in hepatocytes involves endoplasmic reticulum stress, mitochondrial dysfunction and oxidative stress. ToxicolApplPharmacol, 265(1), 73-82.

26. Motawi, T.M., Sadik, N.A. and Refaat, A.2010. Cytoprotective effects of DL alpha-lipoic acid or squalene on cyclophosphamide-induced oxidative injury: an experimental study on rat myocardium, testicles and urinary bladder. Food Chem Toxicol.;48(8-9): 2326-2336.

27. Muhammed, M.H., Randa, M., Mostafa, R.M., Said, M.A., Elgndy, A.I. and Elwakel H.E. 2021.Protective Effect of Thymoquinone on Bisphenol A-Induced Hepatotoxicity in Male Rats, Targeting the Role of Associated Pro-Inflammatory Cytokines and NF-kB. BMFJ;38 (academic issue): 61-72.

28. Murata, M., Suzuki, T., Midorikawa, K., Oikawa, S. and Kawanishi, S.2004. Oxidative DNA damage 
induced by a hydroperoxide derivative of cyclophosphamide. Free RadicBiol Med. 2004;37(6): 793-802.

29. Nabavi, S. F., Daglia, M., Moghaddam, A. H., Habtemariam, S., and Nabavi, S. M. 2014. Curcumin and liver disease: from chemistry to medicine. Compr.Rev. Food Sci. Food Saf. 13, 62-77.

30. Paglia, D.E. and Valentine W. N. 1967. Studies on the quantitative and qualitative characterization of erythrocyte glutathione peroxidase. J. Lab. Clin.Med. 70: $158-169$.

31. Paniagua, A.C. and Amariles, P. 2017. Hepatotoxicity by drugs.and Adverse Effects of Drugs - Mechanisms and Risks Factors, NtambweMalangu, IntechOpen.

32. Priyadarsini, K.I., Maity, D.K., Naik, G.H., Kumar, M.S., Unnikrishnan, M.K., Satav, J.G. and Mohan, H .2003.Role of phenolic O-H and methylene hydrogen on the free radical reactions and antioxidant activity of curcumin. Free RadicBiol Med 35:475-484.

33. Rafiee, Z., Nejatian, M., Daeihamed, M., and Jafari, S. M. 2019.Application of different nanocarriers for encapsulation of curcumin. Crit. Rev. Food Sci. Nutr. 59, 3468-3497.

34. Ranjbar, A., Gholami, L., Ghasemi, H., and Kheiripour, N. 2020.Effects of nanocurcumin and curcumin on the oxidant and antioxidant system of the liver mitochondria in aluminum phosphide-induced experimental toxicity. Nanomed. J. 7, 58-64.

35. Reddy, A.C.P. and Lokesh, B.R. 1994. Alterations in lipid peroxides in rat liver by dietary $n-3$ fatty acids: modulation of antioxidant enzymes by curcumin, eugenol, and vitamin E. J NutrBiochem 5:181-188.

36. Rudramurthy, G., Swamy, M., Sinniah, U. and Ghasemzadeh, A. 2016. Nanoparticles: alternatives against drug-resistant pathogenic microbes. Molecules 21,836 .
37. Singh, A., Bhat, T.K. and Sharma, O.P. 2011. Clinical Biochemistry of Hepatotoxicity.J Clinic Toxicol. S:4:1-19.

38. Singh, C., Prakash, C., Tiwari, K.N., Mishra, S.K. and Kumar, V. 2018.Premnaintegrifolia ameliorates cyclophosphamide-induced hepatotoxicity by modulation of oxidative stress and apoptosis. Biomed Pharmacother, 107: 634-643.

39. Willenbacher, E., Khan, S. Z., Mujica, S. C. A., Trapani, D., Hussain, S. and Wolf, D. 2019. Curcumin: New Insights into an Ancient Ingredient against Cancer. Int. J. Mol. Sci. 20, 1808.

40. Yallapu, M. M., Jaggi, M., and Chauhan, S. C. 2012.Curcumin nanoformulations: a future nanomedicine for cancer. Drug Discovery Today17, 71-80.

41. Yetkine. Z.A, Bakır,B., Bozkurt, Ş.B., Kayis, S.A. and Hakki, S.S. 2020. Positive effect of curcumin on experimental peridontitis via suppression of IL-1-beta and IL-6 expression level, International Journal for Vitamin and Nutrition Research.

42. Yildiz, S.C., Keskin C., Sahintürk V. and Ayhanci A.2019.A Histopathological, Immunohistochemical and Biochemical Investigation on the in vitro Antioxidant, Myeloprotective, Hematoprotective and Hepatoprotective Effects of Hypericumtriquetrifolium Seed Extract Against Cyclophosphamide-Induced Toxicity. Brazilian Archives of Biology and Technology. Vol.62:1-14.

43. Z. Klin. Chem. Klin. 1972. Recommendations of the German Society for Clinical Chemistry. Standardization of methods for the estimation of enzyme activities in biological fluids. Experimental basis for the optimized standard conditions. Biochem. 10: $281-291$ 